\title{
The effects of oral feeding while on nasal continuous positive airway pressure (NCPAP) in preterm infants
}

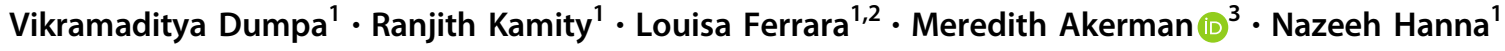

Received: 14 October 2019 / Revised: 30 January 2020 / Accepted: 11 February 2020 / Published online: 21 February 2020

(c) The Author(s), under exclusive licence to Springer Nature America, Inc. 2020

\begin{abstract}
Objective To determine whether delaying oral feeding until coming off NCPAP will alter feeding and respiratory-related morbidities in preterm infants.

Design In this retrospective pre-post analysis, outcomes were compared in two preterm infant groups ( $\leq 32$ weeks gestation). Infants in Group 1 were orally fed while on NCPAP, while infants in Group 2 were only allowed oral feedings after ceasing NCPAP.

Results Although infants in Group 2 started feeds at a later postmenstrual age (PMA), they reached full oral feeding at a similar PMA compared with Group 1. Interestingly, there was a positive correlation between the duration of oral feeding while on NCPAP and the time spent on respiratory support in Group 1.

Conclusions Delayed oral feeding until ceasing NCPAP did not contribute to feeding-related morbidities. We recommend caution when initiating oral feedings in preterm infants on NCPAP without evaluating the safety of the infants and their readiness for oral feedings.
\end{abstract}

\section{Introduction}

It is estimated that $30-70 \%$ of very low birth weight infants (VLBW) are diagnosed with swallowing dysfunction, with an inverse relationship between severity and gestational age (GA) [1-4]. The maturation of suck, swallow, and breathe coordination occurs around 32-34 weeks postmenstrual age (PMA) in infants born prematurely. It is a common practice in neonatal intensive care units (NICUs) to initiate the transition from gavage to oral feeds around this PMA [5-8] since delayed initiation has been linked to numerous medical and developmental consequences, such as delayed oral motor development, prolonged hospital stays, and the persistence of feeding disorders [6,9-12]. On the other

Nazeeh Hanna

nazeehhanna@gmail.com

1 Division of Neonatology, Department of Pediatrics, NYU Winthrop Hospital, 259 First St, Mineola, NY 11501, USA

2 Department of Communication Sciences and Disorders, Molloy College, 100 Hempstead Ave, Rockville Centre, NY 11756, USA

3 Department of Biostatistics, NYU Winthrop Hospital, 101 Mineola Blvd, Mineola, NY 11501, USA hand, the initiation of oral feeding before preterm infants' swallowing mechanisms have become fully mature can lead to aspiration [13, 14]. Chronic aspiration results in a persistent inflammatory state, disease progression, and eventually chronic lung injury $[15,16]$. Such sequelae can be devastating for the already fragile and developmentally immature lungs of VLBW infants. In addition to their immature suck and swallow coordination that prevents them from protecting their airways, aspiration/penetration is often silent and occurs without any clinical evidence of dysphagia (e.g., coughing, choking, or gagging) [3, 17-19], thereby placing this fragile population at even greater risk for aspiration-related complications [2, 20, 21].

Several publications have suggested that aspiration during oral feeding in preterm infants is mainly related to inappropriate swallow-respiration interaction rather than suck-swallow interaction [22, 23]. During their hospital stay, VLBW infants often require respiratory support, including noninvasive ventilation that delivers positive inflation pressure to the airway $[24,25]$, such as nasal continuous positive airway pressure (NCPAP), and high-flow nasal cannulas (HFNC) [26, 27]. However, no clear evidence exists to support the practice of oral feeding while on noninvasive respiratory support, where the positive pressure can potentially interfere with the swallow-respiration interaction. 
Consequently, there exists no consensus or uniform approach among clinicians concerning initiating oral feeds in such situations [28]. Factors that compromise the already immature swallow-respiration interaction in preterm infants have not been adequately investigated. Our previously published data suggested that oral feeding while on NCPAP significantly increased the infants' risk of laryngeal penetration and tracheal aspiration [17]. The pressurized airflow provided by NCPAP likely interfered with the swallowing mechanism in infants on oral feedings, thereby exacerbating the potential of silent aspiration and its resulting consequences. Other recent publications also advised against orally feeding infants on noninvasive ventilation before further safety studies are available [29-32].

Based on our previous results [17], our institution discontinued the practice of orally feeding infants while on NCPAP starting in July 2015. Such change of practice, however, raised a concern that delaying the transition to oral feeding until preterm infants are off NCPAP may result in their delayed achievement of full oral feeding as well as other feeding-related morbidities. In this retrospective pre-post analysis, feeding and respiratory-related outcomes were compared in preterm infants ( $\leq 32$ weeks gestation) before and after discontinuing the practice of oral feeding while on NCPAP in our NICU.

\section{Materials and methods}

This retrospective study compares the outcomes of premature infants born during two periods. All infants at a GA of $\leq 32$ weeks, who were admitted to our Regional Perinatal Center NICU at NYU Winthrop Hospital, were included in this analysis over two periods: "Period 1", from January 2012 to July 2015 (oral feeding allowed while on NCPAP) and "Period 2" from August 2015 to December 2017 (oral feeding prohibited while on NCPAP). Three groups were compared.

Group 1 (Period 1, 2012-2015, $n=39$ ): oral feedings were started when all the following inclusion criteria were met: (a) PMA of $\geq 34$ weeks, (b) on NCPAP, and (c) positive oral feeding cues. Group 2: (Period 2, 2015-2017, $n=60$ ): oral feedings were started when all the following inclusion criteria were met: (a) PMA of $\geq 34$ weeks, (b) off NCPAP, and (c) positive oral feeding cues. In Group 2, since oral feeding was prohibited while on NCPAP, oral feeding was started at a later PMA (compared with Group 1) and included infants that had developed oral feeding cues at earlier PMA (but were never allowed to orally feed because they were on NCPAP) as well as infants that developed oral feeding cues at a later PMA. There was an exclusion bias in Group 1, with some infants being excluded from this group for lack of positive oral feeding cues at earlier PMA. This subgroup had oral feeding started at a later PMA when they later developed oral feeding cues (all of them were off NCPAP by the time oral feeding started). We included this subgroup in the analysis as a separate group (Group 3). Oral feedings were started in Group 3 (Period 1, 2012-2015, $n=10$ ) when all the following inclusion criteria were met: (a) PMA of $\geq 34$ weeks, (b) off NCPAP, and (c) positive oral feeding cues.

The PMA of 34 weeks as the GA to offer oral feeding was the standard clinical practice in our NICU since several publications suggested maturation of the swallow mechanism in preterm infants at this PMA [7, 33, 34]. Our clinical practice incorporates the offering of oral feeds only to those infants who demonstrate cues of oral feeding readiness as assessed by the bedside registered nurses. This objective oral feeding assessment (based on alertness, tone, hunger cues, and latching) was developed by our NICU and was consistently used during the study period (2012-2017). The NCPAP support was ventilator delivered via either Hudson prongs (Hudson-RCI, Temecula, CA) or RAM cannula ${ }^{\oplus}$ (Neotech, Valencia, CA) interfaces during the study period and the positive end-expiratory pressure ranged from 5 to $8 \mathrm{~cm} \mathrm{H}_{2} \mathrm{O}$. During the study period, we did not use highflow nasal cannula in our NICU and the preterm infants were weaned off NCPAP to regular $\mathrm{NC} \leq 21 / \mathrm{min}$ flow or room air. There were no recorded significant practice changes in the management of infants including ventilator strategies, oral feeding protocols, or pre- or postnatal steroid use during this period.

Exclusion criteria included any conditions that necessitated a delay in the initiation or advancement of enteral feedings, such as necrotizing enterocolitis, gastrointestinal disorders, or congenital anomalies, among others. Oral feeding was defined as any amount of milk or formula taken by mouth, while bronchopulmonary dysplasia (BPD) was defined according to the National Institutes of Health consensus definition [35]. Intraventricular hemorrhage (IVH) grading was performed following Papile's classification [36].

Our primary outcomes of interest were the infants' duration to achieve full oral feeding and length of stay. Secondary outcomes included respiratory morbidities, such as BPD, discharge on diuretics, discharge on home oxygen, and other relevant clinical outcomes. In Group $1(n=39)$, we also correlated the duration of exposure to oral feeds while on NCPAP and the time taken to wean off all respiratory support (starting from the day of oral feeding initiation). In this analysis, we included infants ( $n=33$ out of 39) who required fraction of inspired oxygen $\leq 0.25$ at the time of oral feeding initiation to make this group more homogenous in terms of respiratory disease severity. All data were extracted from the electronic medical record, and the medical record review for this study was approved by the hospital's institutional review board. 


\section{Statistical methods}

Descriptive statistics (median, 25th and 75th percentiles for continuous variables; frequencies and percentages for categorical variables) were calculated separately by groups. The chi-square test or Fisher's exact test, as deemed appropriate, for categorical variables and the Mann-Whitney test (two-group comparisons) and the Kruskal-Wallis test (three group comparisons) for continuous data were used to compare the groups. Within Group 1, the correlation between the duration of oral feeds while on NCPAP and the time taken to wean off respiratory support was assessed using the Pearson correlation coefficient.

The analysis of length of stay ("time to discharge alive") was accomplished by applying standard methods of survival analysis, i.e., computing the Kaplan-Meier [37] productlimit curves, where the data were stratified by group. In cases where the endpoint event had not yet occurred, the time until the last follow-up was used and considered "censored." The groups were compared using the log-rank test. The median rates for each group were obtained from the Kaplan-Meier/ product-limit estimates, and their corresponding 95\% confidence intervals were computed using Greenwood's formula to calculate the standard error [38]. A result was considered statistically significant at the $p<0.05$ level of significance. All analyses were performed using SAS version 9.4 (SAS Institute Inc., Cary, NC).

\section{Results}

Infants in all three groups were comparable, and no significant differences were identified in the baseline characteristics of GA, birth weight, antenatal or postnatal steroid use, although a higher female gender in Group 1 and higher cesarean delivery rate in Group 2 was reported (Table 1). No significant differences were determined between the groups regarding the number of days on NCPAP and the number of days on noninvasive ventilation, nasal intermittent positive pressure ventilation, or mechanical ventilation (Table 2). As expected, infants in Group 1 had an earlier initiation of oral feeds (median PMA 35.2 weeks) compared with Group 2 (median PMA 35.8 weeks $p=0.0001$ ) and Group 3 (median PMA 35.9 weeks, $p=0.003$ ). The infants in Group 1 took longer to achieve full oral feeding compared with Groups 2 and 3 (median days 16 vs. 10 vs. $10, p=0.02$ ). The PMA at which infants reached full oral feeding, however, was not significantly different between the groups (median PMA 37.2 weeks vs. 37.6 weeks vs. 37.2 weeks, $p=0.54$, respectively). The length of their NICU stay was also not significantly different between the groups (median days $90 \mathrm{vs}$. 86 vs. $102.5, p=0.41$, respectively)

In an analysis of the complications associated with prematurity (Table 3), no significant differences among the groups were identified, including the patent ductus arteriosus that required ligation, IVH grade 3 or above, periventricular leukomalacia, culture-positive sepsis, or the need for a gastrostomy tube. No difference was identified in the number of infants discharged home on oxygen among the groups. There was no difference found between the groups regarding the overall rate of BPD.

A Pearson correlation coefficient analysis was performed in Group 1 to determine if there was an association between the number of days of oral feeds while on NCPAP and the time required to wean off all respiratory support (wean to room air) starting from the day the oral feeding was initiated. Interestingly, a positive correlation was noted as illustrated in Fig. $1(r=0.63, p<0.0001)$.

\section{Discussion}

This study demonstrates that delaying oral feeding until preterm infants are off NCPAP does not result in feedingrelated morbidities. Furthermore, our results suggest a positive correlation between duration of oral feeding while on NCPAP and the time spent on respiratory support in preterm infants.
Table 1 Demographic characteristics.

\begin{tabular}{lcclc}
\hline & Group 1 $(n=39)$ & Group 2 $(n=60)$ & $\begin{array}{l}\text { Groups 1 vs. 2, } \\
p \text { value }\end{array}$ & Group 3 $(n=10)$ \\
\hline Gestation age (weeks) $^{\mathrm{a}}$ & $27.0(25.6,28.5)$ & $27.5(25.8,28.9)$ & 0.58 & $27.0(25.2,28.0)$ \\
${\text { Birth weight }(\mathrm{g})^{\mathrm{a}}}$ & $820(670,1160)$ & $882.5(755,1175)$ & 0.46 & $820(770,950)$ \\
Female Gender $n(\%)$ & $25(64.1 \%)$ & $25(41.7 \%)$ & 0.03 & $4(40.0 \%)$ \\
Antenatal steroids $n(\%)$ & $36(92.3 \%)$ & $59(98.3 \%)$ & 0.30 & $9(90.0 \%)$ \\
Cesarean delivery $n(\%)$ & $31(79.5 \%)$ & $57(95.0 \%)$ & 0.02 & $7(70.0 \%)$ \\
Postnatal steroids $n(\%)$ & $3(7.7 \%)$ & $11(18.3 \%)$ & 0.14 & $1(10.0 \%)$ \\
\hline
\end{tabular}

${ }^{a}$ Values are shown as medians (interquartile ranges).

${ }^{*} p$ value was not significant when characteristics are compared between Group 3 vs. Group 1 or Group 3 vs. Group 2. 
Table 2 Respiratory and feeding milestones.

Group $1(n=39) \quad$ Group $2(n=60) \quad$ Groups 1 vs. 2, Group $3(n=10)$ $p$ value

\begin{tabular}{lcccc}
\hline $\begin{array}{l}\text { Days on NCPAP } \\
\text { Days on noninvasive } \\
\text { ventilation }\end{array}$ & $28(20,36)$ & $30(20.5,38)$ & 0.65 & $20(16,29)$ \\
$\begin{array}{l}\text { Days on NIPPV } \\
\text { Days on MV }\end{array}$ & $53(41,70)$ & $49.5(41,62)$ & 0.36 & $46(32,56)$ \\
$\begin{array}{l}\text { PMA at oral feeds first } \\
\text { attempted }\end{array}$ & $35.2(34.5,35.6)$ & $35.8(35,37)$ & 0.0001 & $24(5,30)$ \\
$\begin{array}{l}\text { Days to achieve full oral } \\
\text { feeds }\end{array}$ & $16(12,21)$ & $10(7,19)$ & 0.01 & $30(2,42)$ \\
$\begin{array}{l}\text { GA at full oral feeds } \\
\text { Length of stay in days }\end{array}$ & $37.2(36.5,38.2)$ & $37.6(36.5,39.2)$ & 0.30 & $10(8,17)$ \\
\hline
\end{tabular}

${ }^{a}$ Values are shown as medians (interquartile ranges).

${ }^{\mathrm{b}}$ Values are shown as medians (95\% confidence interval).

${ }^{\#} p$ value for Groups 1 vs. 3 comparison is 0.012 . $p$ value was not significant when other characteristics are compared between Group 3 vs. Group 1 or Group 3 vs. Group 2.

\begin{tabular}{lcclc}
\hline & $\begin{array}{l}\text { Group 1 } \\
(n=39)\end{array}$ & $\begin{array}{l}\text { Group 2 } \\
(n=60)\end{array}$ & $\begin{array}{l}\text { Groups 1 vs. 2, } \\
p \text { value }\end{array}$ & $\begin{array}{l}\text { Group 3 } \\
(n=10)\end{array}$ \\
\hline BPD $n(\%)$ & $28(71.8 \%)$ & $47(78.3 \%)$ & 0.46 & $8(80.0 \%)$ \\
PDA needing ligation $n(\%)$ & $1(2.6 \%)$ & $1(1.7 \%)$ & 1.00 & $1(10.0 \%)$ \\
IVH grade $\geq 3 n(\%)$ & $2(5.1 \%)$ & $9(15 \%)$ & 0.47 & $2(20.0 \%)$ \\
PVL $n(\%)$ & $1(2.6 \%)$ & $1(1.7 \%)$ & 1.00 & $0(0.0 \%)$ \\
Sepsis $n(\%)$ & $3(7.7 \%)$ & $10(16.7 \%)$ & 0.20 & $1(10.0 \%)$ \\
Gastrostomy tube $n(\%)$ & $1(2.6 \%)$ & $2(3.4 \%)$ & 1.00 & $1(10.0 \%)$ \\
Discharge home on diuretics $n(\%)$ & $4(10.2)$ & $1(1.6)$ & 0.056 & $0(0.0 \%)$ \\
Discharge home on oxygen $n(\%)$ & $3(7.6)$ & $1(1.6)$ & 0.14 & $1(10.0 \%)$ \\
\hline
\end{tabular}

${ }^{\#} p$ value was not significant when characteristics are compared between Group 3 vs. Group 1 or Group 3 vs. Group 2.

Table 3 Morbidity characteristics.

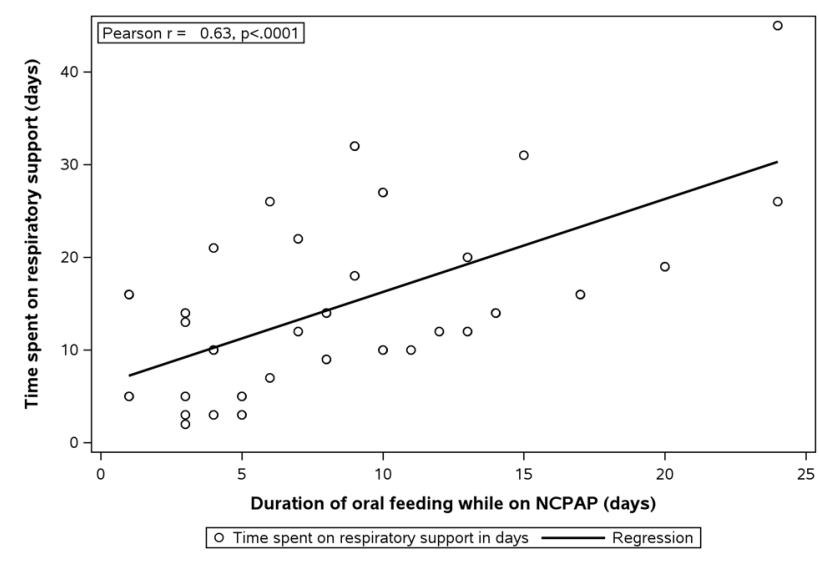

Fig. 1 Infants in Group 1 with the duration of oral feeding while on NCPAP plotted on the $x$-axis and days on respiratory support starting from oral feed initiation plotted on the $\boldsymbol{y}$-axis. A positive correlation was noted $(r=0.63, p<0.0001)$.
Very scant literature supports or refutes the practice of initiating oral feeding in infants on NCPAP. A high degree of coordination between the sensorimotor sequences of respiration and swallowing is required for safe oral ingestion [39], which is often inadequate in infants born preterm due to physiologic instability, immature neurologic systems, and underdeveloped cardiorespiratory systems $[2,14,40,41]$. Risk factors that further compromise this already immature swallowing mechanism in preterm infants have rarely been evaluated. The use of NCPAP is reported to induce the dilatation of the laryngeal opening in preterm infants and might interfere with the sensory reception of the liquid bolus [42] as well as inhibit the swallow reflex in adults [43]. Our previous publication demonstrated that oral feeding while on NCPAP significantly increased the risk of penetration/aspiration events in preterm infants, as demonstrated by the videofluoroscopic swallow study [17]. The theoretical rationale 
for this finding was that the presence of pressurized airflow provided by the NCPAP interfered with the sensory reception of the liquid bolus. This interference resulted in altered sensorimotor sequences of the pharyngeal swallowing mechanism, causing a dysfunctional swallow with airway compromise.

Samson et al. [29] reported an increased incidence of coughing in lambs on NCPAP immediately following bottle feeding (27\% in NCPAP group vs. $2 \%$ in controls), which the authors assume to be related to a high milk flow; however, this might also indicate an increased risk of airway compromise with NCPAP, as evidenced by the reflex coughing. The authors concluded that further studies that evaluate tracheal aspirations while feeding under NCPAP are needed. Another study by Hanin et al. [27] demonstrated that infants with BPD who were fed orally while on NCPAP achieved full feeds faster than those infants who were not fed orally. Although the authors implied the safety of this practice, the study solely involved a chest x-ray to exclude the possibility of aspiration pneumonia, and no direct assessment of airway safety was performed. The implied safety is therefore not supported, as a chest $\mathrm{x}$-ray in pediatrics is not sufficiently sensitive for detecting chronic microaspiration due to dysphagia [44, 45]. Furthermore, the study power was not designed to detect differences in respiratory outcomes.

Hori et al. [46] found that CPAP altered the swallow/ respiration cycle in normal, stable adults, thereby increasing their risk of experiencing aspiration. Interestingly, the safety insert in the CPAP commercial machines [47] that are sold to adult patients contains the following warning: "Do not eat or drink while using CPAP. You are likely to inhale the food or drink into your lungs." In spite of the possible detrimental effects of oral feeding during noninvasive ventilation that creates high pressure, the use of such devices (NCPAP and HFNC) in preterm infants during oral feedings is a common practice in many NICUs [25, 27, 48]. Silent aspiration can cause continued inflammation, thus necessitating the requirement for additional respiratory support. Our current retrospective pre-post analysis supports such a risk. In a recent review, experts in the field concluded that the initiation of oral feeds while on noninvasive ventilation can be detrimental, and it is prudent that the routine initiation of oral feeding is avoided while on noninvasive ventilation [30]. However, it has been acknowledged that no published studies guide such practice regarding this important clinical dilemma [30]. In our study, there were no significant differences in respiratory outcomes between groups likely because of the limited sample size.

Delaying oral feeding while on NCPAP is a challenging choice since some neonatologists are concerned about the risk of these infants developing an aversion to oral feeding.
In our current study, we found that, while oral feeding was initiated later in Group 2 (median PMA 35.2 weeks vs. 35.8 weeks, $p=0.0001$ ), there was no significant difference in the PMA at which full oral feeding was achieved (median PMA 37.2 weeks vs. 37.6 weeks, $p=0.30$ ). This finding suggests that oral aversion is not a concern. Furthermore, the fact that both groups reached full oral feeding at the same PMA may suggest that the suck-swallow maturation at a specific PMA is a more important determining factor for successful oral feeding than is the introduction of oral feeding at an early PMA, as demonstrated in other studies [49, 50]. This was enforced by the results of Group 3 (Table 2), as infants in this group reached full oral feeding at a similar PMA (37.2 weeks) compared with Group 1, in spite of later initiation of oral feeding (35.9 weeks). This finding may also suggest that infants in Group 1 took a longer time to achieve full feeds due to the altered sensorymotor environment caused by the NCPAP. Infants in Groups 2 and 3 learned to feed under a more typical sensory-motor feeding environment, and therefore learned more proficiently. In addition, no difference was identified in the length of stay between the groups, thereby indicating that withholding the initiation of oral feeding while on NCPAP did not delay the patients' discharge to home. Interestingly, the outcome measures in Group 3 (Period 1) were similar to Group 2 (Period 2), likely because infants in both groups did not orally feed while on NCPAP.

There are several limitations to the current study. Because it is retrospective in nature, several other factors may have influenced the outcomes. However, there were no significant clinical practice changes recorded in the respiratory or nutritional management of infants within the study period except as mentioned above. We used a ventilator-derived, variable-flow NCPAP system in the current study, for which the findings may not apply to those infants on other types of NCPAP, such as infant flow systems. The differential effect on the outcomes regarding breast milk versus formula was not studied. Also, because our studies were conducted on preterm infants $\leq 32$ weeks GA, which is a group with the most immature suck and swallow mechanisms, the results may not apply to other GA infants. We acknowledge that having a small sample size increases the margin of error and the likelihood that a type II error will skew the results and thereby decrease the study's power. However, data from this study can be used to design larger confirmatory studies. It is also important to highlight that this study does not suggest that oral feeding on NCPAP should not be attempted in all infants $\leq 32$ weeks gestation. It might be appropriate to allow a selected group of preterm infants to feed while on NCPAP orally, but only after implementing reliable and objective testing that assesses the swallowing-breathing interaction to document the safety of and infants' readiness for oral feedings. 
In conclusion, feeding preterm infants orally while on NCPAP does not lead to faster maturation of oral feeding ability, or decreased length of stay. Further research is needed to establish evidence-based oral feeding protocols for preterm infants that include reliable testing for the safety and infants' readiness for oral feedings while on NCPAP.

Acknowledgements Department of Pediatrics, NYU Winthrop Hospital.

Author contributions VD, RK, LF, and NH conceptualized the study, VD and RK performed data collection, and MA performed the statistical analysis. All authors reviewed, revised, and approved the manuscript.

\section{Compliance with ethical standards}

Conflict of interest The authors declare that they have no conflict of interest.

Publisher's note Springer Nature remains neutral with regard to jurisdictional claims in published maps and institutional affiliations.

\section{References}

1. Rommel N, De Meyer AM, Feenstra L, Veereman-Wauters G. The complexity of feeding problems in 700 infants and young children presenting to a tertiary care institution. J Pediatr Gastroenterol Nutr. 2003;37:75-84.

2. Lee JH, Chang YS, Yoo HS, Ahn SY, Seo HJ, Choi SH, et al. Swallowing dysfunction in very low birth weight infants with oral feeding desaturation. World J Pediatr. 2011;7:337-43.

3. Lemons PK. From gavage to oral feedings: just a matter of time. Neonatal Netw. 2001;20:7-14.

4. Field D, Garland M, Williams K. Correlates of specific childhood feeding problems. J Paediatr Child Health. 2003;39:299-304.

5. Lau C, Sheena HR, Shulman RJ, Schanler RJ. Oral feeding in low birth weight infants. J Pediatr. 1997;130:561-9.

6. Breton S, Steinwender S. Timing introduction and transition to oral feeding in preterm infants: current trends and practice. Newborn Infant Nurs Rev. 2008;8:153-9.

7. Mizuno K, Ueda A. The maturation and coordination of sucking, swallowing, and respiration in preterm infants. J Pediatr. 2003; 142:36-40.

8. Medhurst A. Feeding protocols to improve the transition from gavage feeding to oral feeding in healthy premature infants: a systematic review. Adelaide, S. Australia: Australia: Joanna Briggs Institute for Evidence Based Nursing and Midwifery. Health Care Reports; 2005.

9. Ross ES, Browne JV. Feeding outcomes in preterm infants after discharge from the neonatal intensive care unit (NICU): a systematic review. Newborn Infant Nurs Rev. 2013;13:87-93.

10. Simpson C, Schanler RJ, Lau C. Early introduction of oral feeding in preterm infants. Pediatrics. 2002;110:517-22.

11. Crowe L, Chang A, Wallace K. Instruments for assessing readiness to commence suck feeds in preterm infants: effects on time to establish full oral feeding and duration of hospitalisation. Cochrane Database Syst Rev. 2016;CD005586.

12. McGrath JM, Braescu AV. State of the science: feeding readiness in the preterm infant. J Perinat Neonatal Nurs. 2004;18:353-68. quiz $69-70$.
13. Thoyre SM, Holditch-Davis D, Schwartz TA, Melendez Roman CR, Nix W. Coregulated approach to feeding preterm infants with lung disease. Nurs Res. 2012;61:242-51.

14. Davis NL, Liu A, Rhein L. Feeding immaturity in preterm neonates: risk factors for oropharyngeal aspiration and timing of maturation. J Pediatr Gastroenterol Nutr. 2013;57:735-40.

15. Hu X, Lee JS, Pianosi PT, Ryu JH. Aspiration-related pulmonary syndromes. Chest. 2015;147:815-23.

16. Boesch RP, Wood RE. Aspiration. In: Kendig \& Chernick's Disorders of the Respiratory Tract in Children. Philadelphia: Elsevier Saunders; 2012, pp. 947-56.

17. Ferrara L, Bidiwala A, Sher I, Pirzada M, Barlev D, Islam S, et al. Effect of nasal continuous positive airway pressure on the pharyngeal swallow in neonates. J Perinatol. 2017;37:398-403.

18. Ferrara L, Kamity R, Islam S, Sher I, Barlev D, Wennerholm L, et al. Short-term effects of cold liquids on the pharyngeal swallow in preterm infants with dysphagia: a pilot study. Dysphagia. 2018;33:593-601.

19. Linden P, Siebens AA. Dysphagia: predicting laryngeal penetration. Arch Phys Med Rehabil. 1983;64:281-4.

20. Newman LA, Keckley C, Petersen MC, Hamner A. Swallowing function and medical diagnoses in infants suspected of Dysphagia. Pediatrics. 2001;108:E106.

21. Uhm KE, Yi SH, Chang HJ, Cheon HJ, Kwon JY. Videofluoroscopic swallowing study findings in full-term and preterm infants with dysphagia. Ann Rehabil Med. 2013;37:175-82.

22. Lau C, Smith EO, Schanler RJ. Coordination of suck-swallow and swallow respiration in preterm infants. Acta Paediatr. 2003;92:721-7.

23. Pados BF, Thoyre SM, Estrem HH, Park J, Knafl GJ, Nix B. Effects of milk flow on the physiological and behavioural responses to feeding in an infant with hypoplastic left heart syndrome. Cardiol Young. 2017;27:139-53.

24. Mahmoud RA, Roehr CC, Schmalisch G. Current methods of non-invasive ventilatory support for neonates. Paediatr Respir Rev. 2011;12:196-205.

25. Schmolzer GM, Kumar M, Pichler G, Aziz K, O’Reilly M, Cheung PY. Non-invasive versus invasive respiratory support in preterm infants at birth: systematic review and meta-analysis. BMJ. 2013;347:f5980.

26. Glackin SJ, O'Sullivan A, George S, Semberova J, Miletin J. High flow nasal cannula versus NCPAP, duration to full oral feeds in preterm infants: a randomised controlled trial. Arch Dis Child Fetal Neonatal Ed. 2017;102:F329-32.

27. Hanin M, Nuthakki S, Malkar MB, Jadcherla SR. Safety and efficacy of oral feeding in infants with BPD on nasal CPAP. Dysphagia. 2015;30:121-7.

28. Canning A, Fairhurst R, Chauhan M, Weir KA. Oral feeding for infants and children receiving nasal continuous positive airway pressure and high-flow nasal cannula respiratory supports: a survey of practice. Dysphagia. 2019. https://doi.org/10.1007/s00455019-10047-4 [Epub ahead of print].

29. Samson N, Michaud A, Othman R, Nadeau C, Nault S, Cantin D, et al. Nasal continuous positive airway pressure influences bottlefeeding in preterm lambs. Pediatr Res. 2017;82:926-33.

30. Jadcherla SR, Bhandari V. "Pressure" to feed the preterm newborn: associated with "positive" outcomes? Pediatr Res. 2017;82:899-900.

31. Leder SB, Siner JM, Bizzarro MJ, McGinley BM, Lefton-Greif MA. Oral alimentation in neonatal and adult populations requiring high-flow oxygen via nasal cannula. Dysphagia. 2016;31:154-9.

32. Dodrill P, Gosa M, Thoyre S, Shaker C, Pados B, Park J, et al. First, do no harm: a response to "oral alimentation in neonatal and adult populations requiring high-flow oxygen via nasal cannula". Dysphagia. 2016;31:781-2.

33. Bu'Lock F, Woolridge MW, Baum JD. Development of coordination of sucking, swallowing and breathing: ultrasound study of term and preterm infants. Dev Med Child Neurol. 1990;32:669-78. 
34. Medoff-Cooper B, McGrath JM, Bilker W. Nutritive sucking and neurobehavioral development in preterm infants from 34 weeks PCA to term. MCN Am J Matern Child Nurs. 2000;25:64-70.

35. Ehrenkranz RA, Walsh MC, Vohr BR, Jobe AH, Wright LL, Fanaroff AA, et al. Validation of the National Institutes of Health consensus definition of bronchopulmonary dysplasia. Pediatrics. 2005;116:1353-60.

36. Papile LA, Burstein J, Burstein R, Koffler H. Incidence and evolution of subependymal and intraventricular hemorrhage: a study of infants with birth weights less than 1,500 gm. J Pediatr. 1978;92:529-34.

37. Lee ET. Statistical methods for survival data analysis. 2nd ed. New York: John Wiley \& Sons; 1992.

38. Greenwood M. The errors of sampling of the survivorship table, vol. 33 of reports on public health and medical subjects. London: Her Majesty's Stationery Office; 1926.

39. Nishino T. The swallowing reflex and its significance as an airway defensive reflex. Front Physiol. 2012;3:489.

40. Thoyre SM, Shaker CS, Pridham KF. The early feeding skills assessment for preterm infants. Neonatal Netw. 2005;24:7-16.

41. Goldfield EC, Wolff PH, Schmidt RC. Dynamics of oralrespiratory coordination in full-term and preterm infants: I. Comparisons at 38-40 weeks postconceptional age. Dev Sci. 1999;2:363-73.

42. Gaon P, Lee S, Hannan S, Ingram D, Milner AD. Assessment of effect of nasal continuous positive pressure on laryngeal opening using fibre optic laryngoscopy. Arch Dis Child Fetal Neonatal Ed. 1999;80:F230-2.

43. Nishino T, Sugimori K, Kohchi A, Hiraga K. Nasal constant positive airway pressure inhibits the swallowing reflex. Am Rev Respir Dis. 1989;140:1290-3.

44. Lefton-Greif MA, Carroll JL, Loughlin GM. Long-term follow-up of oropharyngeal dysphagia in children without apparent risk factors. Pediatr Pulmonol. 2006;41:1040-8.

45. Miller CK. Feeding issues and interventions in infants and children with clefts and craniofacial syndromes. Semin Speech Lang. 2011;32:115-26.

46. Hori R, Isaka M, Oonishi K, Yabe T, Oku Y. Coordination between respiration and swallowing during non-invasive positive pressure ventilation. Respirology. 2016;21:1062-7.

47. Respicair PC. Tips for using CPAP/BIPAP. Niagara Falls, NY: Respicair PC; 2019. http://respicairpc.com/cpap-and-bipap-tips/.

48. Baraldi E, Filippone M. Chronic lung disease after premature birth. N Engl J Med. 2007;357:1946-55.

49. Pickler RH, Reyna BA, Wetzel PA, Lewis M. Effect of four approaches to oral feeding progression on clinical outcomes in preterm infants. Nurs Res Pract. 2015;2015:716828.

50. White-Traut R, Pham T, Rankin K, Norr K, Shapiro N, Yoder J. Exploring factors related to oral feeding progression in premature infants. Adv Neonatal Care. 2013;13:288-94. 